\title{
Determination of the Anti-Adhesive and Anti-Biofilm Capacity of a Wheat Extract on Staphylococcus aureus in Farms
}

\author{
Maria Angeles Calvo Torras' ${ }^{1}$, Esteban Leonardo Arosemena Angulo', \\ Susana Martin Orúe ${ }^{2}$, Xavier Torras Bombardó ${ }^{1}$ \\ ${ }^{1}$ Universitat Autònoma de Barcelona (UAB), Facultad de Veterinaria, Department of Animal health and Anatomy, \\ Cerdanyola del Vallès, Spain \\ ${ }^{2}$ Universitat Autònoma de Barcelona (UAB), Facultad de Veterinaria, Department of Animal and Food Science, \\ Cerdanyola del Vallès, Spain \\ Email: mariangels.calvo@uab.cat
}

How to cite this paper: Calvo Torras, M.A., Arosemena Angulo, E.L., Martin Orúe, S. and Torras Bombardó, X. (2021) Determination of the Anti-Adhesive and Anti-Biofilm Capacity of a Wheat Extract on Staphylococcus aureus in Farms. Journal of Materials Science and Chemical Engineering, 9, 11-21.

https://doi.org/10.4236/msce.2021.99002

Received: July 26, 2021

Accepted: September 23, 2021

Published: September 26, 2021

Copyright $\odot 2021$ by author(s) and Scientific Research Publishing Inc. This work is licensed under the Creative Commons Attribution International License (CC BY 4.0).

http://creativecommons.org/licenses/by/4.0/

\begin{abstract}
The formation of biofilm by pathogenic microorganisms has become a problem in the livestock industry since it is considered a potential source of infection for farm animals while increasing microbial resistance to physical and chemical agents. Some plant extracts, such as soluble wheat extract, have been shown to be effective in inhibiting or destroying the biofilm of certain microorganisms under specific conditions. The objective of this study is to evaluate the capacity of the pathogen to form biofilm on different surfaces used in livestock, as well as to evaluate the anti-biofilm capacity of the soluble wheat extract against $S$. aureus on these surfaces. The inhibition potential of inhibition or destruction of biofilm was tested in vitro. Wheat extract at a concentration of $0.29 \mathrm{mg} / 100 \mathrm{~mL}$ showed anti-biofilm activity on $S$. aureus, inhibiting its formation, as well as destroying it greatly after a contact time of 24 hours, on those surfaces where the microorganism presents more adhesion capacity.
\end{abstract}

\section{Keywords}

Microbiology, Biofilm, Anti-Biofilm, Wheat Extract, Staphylococcus Aureus

\section{Introduction}

Microbial contamination and infections have always been a problem and a limiting factor for growth and animal production on fattening farms. To combat and prevent certain livestock infections by certain microorganisms, antimicrobial therapy has been used in animals in recent years. Even so, several studies question 
this methodology due to the increasingly frequent appearance of resistance to these compounds. Therefore, it is necessary to find new ways to prevent microbial infections in livestock that are independent of substances that can generate resistance [1]. Several studies have proposed alternative products that can be used as additives in animal feed, with the ability to prevent adhesion and intestinal colonization of pathogenic bacteria in animals, which also do not generate resistance by microorganisms [2] [3] [4].

Some of the alternative products can be such as prebiotics, probiotics, plant extracts, organic acids, enzymes or microminerals.

A different problem from colonization and infection of cattle is the ability of certain microorganisms to adhere to certain types of surfaces, forming films of microbial communities enveloped by an extracellular matrix called "biofilms". It is known that microbial growth in nature is found mainly in the form of biofilms since in this way the microorganisms present more resistance to environmental factors and antimicrobial agents than in the free form [5]. In some cases, low doses of antibiotics may even favour the formation and growth of a biofilm, which indicates a natural defence mechanism for microorganisms to avoid the toxic effect of these substances [6]. These structures are therefore much more difficult to combat by physical or chemical methods and can cause livestock infections more easily [7].

Some natural products have been shown to be effective against the adhesion of certain microorganisms to surfaces, thus presenting new study possibilities to combat the formation of biofilms in fattening farms independently of microbial substances [8] [9]. Even so, many of these substances or natural extracts have complex compositions and it is, therefore, difficult to determine their activity and their ability to block the adhesion of bacterial cells to surfaces is in some cases purely empirical [10].

In this work, the ability of a natural extract from rye grain to inhibit the formation and/or break biofilm of the pathogenic microorganism Staphylococcus aureus on different types of surfaces of snail and poultry farms has been tested. The efficacy of this natural extract was previously described against biofilms formed in epithelial tissue of mammary glands in cows, causing mastitis and subclinical infections of the mammary glands [10].

$S$. aureus is a non-sporulated facultative anaerobic microorganism that is Gram-positive cocci and is positive for catalase and coagulase reactions. Despite being a pathogenic microorganism, it is widely distributed in a wide variety of environments and habitats, also forming part of the normal microbiota of the skin and mucosa of warm-blooded animals. It can cause a wide variety of infections and diseases, either through infection and colonization of tissues, or through the production of toxins, among which several types of enterotoxins and haemolysins stand out. Among the diseases caused by this microorganism, stand out from benign mucosal and skin infections or gastroenteritis caused by enterotoxins, to infections of internal tissues, pneumonia, meningitis, endocarditis or 
sepsis, among others.

The adhesion of $S$. aureus on animal tissue surfaces and the formation of biofilms have been described as important virulence factors [3]. It is also known that the mechanism described as "quorum sensing" intervenes in the regulation and coordination between the different microorganisms responsible for the creation and maintenance of a biofilm [11] [12]. In this sense, the plant extract tested in this study has been shown to be a potential inhibitor of biofilm formation due to its anti-adhesive properties against Enterobacteriaceae [1] [13] [14].

The main objective of the work is to demonstrate the effect that a soluble wheat extract has on $S$. aureus biofilm on four different types of surfaces:

- A PVC surface, a metal surface and a cloth surface are used as a coating for rooms on snails farms.

- Plastic material channelling tube is used to channel the water used in poultry farm waterers.

For all surfaces, the ability of the wheat extract to prevent the formation of $S$. aureus biofilm was determined, as well as the ability to destroy an already formed biofilm and prevent its reappearance.

We select wheat extract because is very easy for us their obtention.

\section{Material and Methods}

To carry out the different tests, the 4 surfaces were divided into strictly equal parts as follows:

- PVC, metallic and cloth material used in the snails farm: Different portions of $6 \times 7 \mathrm{~cm}$ each were made, previously disinfected with ethanol (96\%).

- Material used in the poultry farm: The channelling tube was cut into $8 \mathrm{~cm}$ portions, and then sterilized at $121^{\circ} \mathrm{C}$ for 20 minutes.

For all the tests carried out, an overnight culture of the microorganism was started in rich medium BHI (Brain heart infusion).

The process was carried out at all times under sterile conditions to avoid contamination of the samples. As a diluent for the preparation of the different samples, a sterile solution of $50 \mathrm{~g} / \mathrm{L}$ glucose and $9 \mathrm{~g} / \mathrm{L} \mathrm{NaCl}$ was used to simulate the conditions of the poultry farm, to which $3 \mathrm{~g} / \mathrm{L}$ of a supplement of amino acids and vitamins for birds after sterilization.

All tests were made at the same concentration of wheat extract $(0.29 \mathrm{mg} /$ $100 \mathrm{~mL}$ of solution). We select this concentration because is the best for price and system

\subsection{Determination of the Extract's Ability to Prevent the Formation of $S$. aureus Biofilm}

To determine the inhibitory capacity of biofilm formation, $100 \mathrm{~mL}$ of each of the following sterile dilutions were prepared:

- Positive control: $50 \mathrm{~mL}$ of the glucose saline solution to which $0.3 \mathrm{~g}$ of the amino acid and vitamin supplement was added with $50 \mathrm{~mL}$ of the overnight 
culture of the microorganism.

- Negative control: $100 \mathrm{~mL}$ of the glucose saline solution to which $0.3 \mathrm{~g}$ of the amino acid and vitamin supplement with $0.29 \mathrm{mg}$ of wheat extract were added.

- Sample: $50 \mathrm{~mL}$ of the glucose saline solution to which $0.3 \mathrm{~g}$ of the amino acid and vitamin supplement was added with $50 \mathrm{~mL}$ of the overnight culture of the microorganism and with $0.29 \mathrm{mg}$ of wheat extract.

\subsubsection{Surfaces Supplied by the Snails Farm}

The surface was divided into 15 portions of $6 \times 7 \mathrm{~cm}$, in which $50 \mu \mathrm{L}$ of the following dilutions were inoculated:

- "Positive control" in 3 portions.

- "Negative control" in 3 portions.

- "Sample" in 9 portions.

The $50 \mu \mathrm{L}$ of each dilution was spread homogeneously by each portion using a sterile Digralsky loop.

A sample (see Section 2.3) was taken from the portions identified as positive control, negative control and 3 replicates of the "sample" solution at 0,24 and 48 $\mathrm{h}$ after the initial inoculation. During the periods between the sampling, the surface was incubated at $37^{\circ} \mathrm{C}$ under aerobic conditions.

\subsubsection{Pipes from Poultry Farm}

Twelve portions of pipes $(8 \mathrm{~cm} /$ portion) were inoculated with the following solutions:

- 3 portions with the "Positive Control" solution.

- 3 portions with the "Negative Control" solution.

- 6 portions with the "Sample" solution.

A sample of the liquid and the surface (see Section 2.3.) were taken from the positive and negative controls and 3 replicates of the "sample" solution at 0,24 and $48 \mathrm{~h}$ after the initial inoculation. During the periods between sampling, the tubes were incubated (with the corresponding solution inside) at $37^{\circ} \mathrm{C}$ under aerobic conditions.

\subsection{Determination of the Ability of Wheat Extract to Lysate an Already Formed $S$, aureus Biofilm}

For the determination of the ability of the wheat extract to lyse an already formed biofilm of $S$. aureus, $100 \mathrm{~mL}$ of each of the following sterile dilutions were prepared:

- Initial inoculum: $50 \mathrm{~mL}$ of glucose saline to which $0.3 \mathrm{~g}$ of the amino acid and vitamin supplement was added with $50 \mathrm{~mL}$ of the microorganism overnight culture.

- Positive control: $100 \mathrm{~mL}$ of glucose saline solution to which $0.3 \mathrm{~g}$ of the amino acid and vitamin supplement was added.

- Negative control: $100 \mathrm{~mL}$ of glucose saline solution to which $0.3 \mathrm{~g}$ of the amino acid and vitamin supplement with $0.29 \mathrm{mg}$ of wheat extract were added. 
- Sample: $100 \mathrm{~mL}$ of the glucose saline solution to which $0.3 \mathrm{~g}$ of the amino acid and vitamin supplement with $0.29 \mathrm{mg}$ of wheat extract were added.

\subsubsection{Surfaces Supplied by the Snails Farm}

The surfaces were divided into 10 equal portions of $6 \times 7 \mathrm{~cm}$, in each of which $50 \mu \mathrm{L}$ of the following dilutions were inoculated:

- "Initial inoculum" in all portions at initial time except those corresponding to the negative control.

- "Positive control" in 2 portions on time $24 \mathrm{~h}$.

- "Negative control" in 2 portions at initial time.

- "Sample" in 6 portions and the two portions inoculated with "Negative control", on time $24 \mathrm{~h}$.

The $50 \mu \mathrm{L}$ of each solution was spread evenly over each portion with a sterile Digralsky handle.

A sample (see Section 2.3) was taken from the positive control, negative control, and 3 replicates of the "sample" solution at the initial time and at $24 \mathrm{~h}$ after inoculation on the biofilm previously formed from the "initial inoculum" solution for $24 \mathrm{~h}$. During the periods between inoculations and sampling, the surface was incubated at $37^{\circ} \mathrm{C}$ under aerobic conditions.

\subsubsection{Pipes from Poultry Farm}

Eight $8 \mathrm{~cm}$ tubes were inoculated with the following solutions:

- "Initial inoculum" in all portions at initial time except those corresponding to the negative control.

- "Positive control" in 2 tubes on time $24 \mathrm{~h}$.

- "Negative control" in 2 tubes on time $24 \mathrm{~h}$

- "Sample" in 4 tubes on time $24 \mathrm{~h}$.

A sample of the liquid and the surface (see Section 2.3.) Of the positive control, negative control, and 2 replicates of the "sample" solution were taken at the initial time and $24 \mathrm{~h}$, after inoculation of the controls and samples. During the periods between inoculations and sample collection, the tubes were incubated at $37^{\circ} \mathrm{C}$ under aerobic conditions. For the formation of the biofilm with the "initial inoculum" solution during the first $24 \mathrm{~h}$, the tubes were emptied and incubated dry, while for the following $24 \mathrm{~h}$ they were incubated with the corresponding solutions inside.

\subsection{Sampling}

\subsubsection{On Surfaces Supplied by the Snail Farm}

Sampling on these surfaces was carried out using sterile swabs moistened with $100 \mu \mathrm{L}$ of sterile Ringer's solution in order to collect the maximum number of viables present in each portion. Subsequently, the swab was suspended in a tube with $9 \mathrm{~mL}$ of Ringer's and $100 \mu \mathrm{L}$ were seeded in 3 plates of Mannitol-Salt Agar ( 3 replicates per time and portion). The plates were incubated at $37^{\circ} \mathrm{C}$ for $48 \mathrm{~h}$ under aerobic conditions before proceeding to the colony count. 


\subsubsection{In Tubes Supplied by the Poultry Farm}

1) From the liquid inside the tubes

The liquid was decanted into a sterile tube so as not to affect the number of viables on the surface during handling, it was homogenized and $100 \mu \mathrm{L}$ was used to make the corresponding dilutions with sterile Ringer.

The two most suitable dilutions were chosen to enable a representative and reliable CFU count. From both dilutions, $100 \mu \mathrm{L}$ were seeded in two plates of Mannitol-Salt Agar for each dilution. The plates were incubated at $37^{\circ} \mathrm{C}$ for $48 \mathrm{~h}$ under aerobic conditions before proceeding to the colony count.

2) Of the surfaces of the interior of the tubes

Subsequent to the vacuum of the liquid inside the tubes, a sterile swab was humidified with $100 \mu \mathrm{L}$ of sterile Ringer, and it was passed over the entire surface of the inside of the tubes in order to take the maximum number of viable. Subsequently, the swab was suspended in a tube with $9 \mathrm{~mL}$ of sterile Ringer, from which a serial dilution bank with sterile Ringer was performed. The two most suitable dilutions were chosen to enable a representative and reliable CFU count of both dilutions, $100 \mu \mathrm{L}$ were seeded in two plates of Mannitol-Salt Agar for each dilution. The plates were incubated at $37^{\circ} \mathrm{C}$ for $48 \mathrm{~h}$ under aerobic conditions before proceeding to the colony count.

\section{Results}

\subsection{PVC Surface Supplied by Snails Farm}

The results of the PVC surface used on the snails farm are shown in Table 1. The results are expressed in CFU/unit of surface sampled.

The test of the capacity of the plant extract to inhibit the formation of the biofilm on the PVC surface provided by the snail farm (Table 1), shows an increase in the number of viables of $140.12 \%$ in the samples with respect to the positive control at initial time, while, after 24 and $48 \mathrm{~h}$ of incubation at $37^{\circ} \mathrm{C}$, they present decreases of $62.57 \%$ and $10.50 \%$ respectively with respect to the positive control. Regarding the test in which the capacity of the plant extract to destroy a biofilm on the same surface was evaluated, the counts showed an increase in the number of viable of $12.72 \%$ of the samples over the positive control at initial time, while on time $24 \mathrm{~h}$ they showed a reduction of $58.19 \%$.

Table 1. Counts corresponding to the PVC surface used in the snail farm. The results are expressed in CFU/total area sampled, and the percentage variation of the average of the samples inoculated with the plant extract versus the positive control.

\begin{tabular}{cccccccc}
\hline & $\begin{array}{c}\text { Sample } \\
\text { time (h) }\end{array}$ & $\begin{array}{c}\text { Positive } \\
\text { Control }\end{array}$ & Sample 1 & Sample 2 & Sample 3 & $\begin{array}{c}\text { Average of } \\
\text { 3 samples }\end{array}$ & $\begin{array}{c}\text { Percentage variation } \\
\text { respect to the } \\
\text { positive control }\end{array}$ \\
\hline $\begin{array}{c}\text { Concentration of } \\
\text { microorganism } \\
\text { with the extract }\end{array}$ & 0 & $1.67 \times 10^{4}$ & $1.71 \times 10^{4}$ & $6.99 \times 10^{4}$ & $3.33 \times 10^{4}$ & $4.01 \times 10^{4}$ & $140.12 \%$ \\
\hline $\begin{array}{c}\text { Addition of extract } \\
\text { on biofilm formed }\end{array}$ & 24 & $4.97 \times 10^{3}$ & $2.37 \times 10^{3}$ & $1.67 \times 10^{3}$ & $1.53 \times 10^{3}$ & $1.86 \times 10^{3}$ & $-62.57 \%$ \\
\hline
\end{tabular}




\subsection{Pipes from the Poultry Farm}

The results of the material supplied by the poultry farm for the biofilm inhibition capacity determination test are shown in Table 2 and Table 3. The results are expressed in $\mathrm{CFU} / \mathrm{mL}$ of the liquid contained in each portion and in CFU/unit of sampled area.

When performing the CFU counts per $\mathrm{mL}$ of the liquid contained within each portion, select a reduction of the percentage of viable microorganisms compared to the positive control of $10.75 \%$ at the initial moment, $74.36 \%$ at the $24 \mathrm{~h}$ time and $85.21 \%$ at the time $48 \mathrm{~h}$ after the inoculation of the extract When evaluating the capacity of the extract to destroy a modified biofilm, the affected counts reduced the number of viable of $41.49 \%$ of the samples on the positive control at the initial moment, while at $24 \mathrm{~h}$, it reduced a reduction of $59.62 \%$.

When performing the CFU counts of the interior surface of each portion, a percentage reduction of viable microorganisms was observed compared to the positive control of $36.22 \%$ at initial time, $53.69 \%$ at $24 \mathrm{~h}$ time and $91.00 \%$ at time. It was $91.00 \%$ at $48 \mathrm{~h}$ after inoculation of extract. When evaluating the extract's ability to destroy a formed biofilm, the counts showed a reduction in the number of viable of $41.04 \%$ of the samples over the positive control at initial time, while at $24 \mathrm{~h}$ time they showed a reduction of $95.53 \%$.

\subsection{Metallic Material Supplied by the Snail Farm}

The results of the PVC surface used in the snail farm are shown in Table 4. The

Table 2. Counts corresponding to the liquid inside the tubes used in the poultry farm. The results are expressed in CFU/mL and the percentage variation of the average of the samples inoculated with the plant extract versus the positive control.

\begin{tabular}{|c|c|c|c|c|c|c|}
\hline & $\begin{array}{l}\text { Sample } \\
\text { time (h) }\end{array}$ & $\begin{array}{l}\text { Positive } \\
\text { Control }\end{array}$ & Sample 1 & Sample 2 & $\begin{array}{l}\text { Average of } \\
2 \text { samples }\end{array}$ & $\begin{array}{c}\text { Percentage variation respect } \\
\text { to the positive control }\end{array}$ \\
\hline Concentration of & 0 & $3.35 \times 10^{8}$ & $2.78 \times 10^{8}$ & $3.20 \times 10^{8}$ & $2.99 \times 10^{8}$ & $-10.75 \%$ \\
\hline \multirow{2}{*}{$\begin{array}{l}\text { microorganism } \\
\text { with the extract }\end{array}$} & 24 & $1.17 \times 10^{8}$ & $2.58 \times 10^{7}$ & $3.41 \times 10^{7}$ & $3.00 \times 10^{7}$ & $-74.36 \%$ \\
\hline & 48 & $1.44 \times 10^{7}$ & $2.38 \times 10^{6}$ & $1.88 \times 10^{6}$ & $2.13 \times 10^{6}$ & $-85.21 \%$ \\
\hline on biofilm formed & 24 & $1.08 \times 10^{8}$ & $4.20 \times 10^{7}$ & $4.43 \times 10^{7}$ & $4.36 \times 10^{7}$ & $-59.62 \%$ \\
\hline
\end{tabular}

Table 3. Counts corresponding to the interior surface of the tubes used in the poultry farm. The results are expressed in CFU/area sampled, and the percentage variation of the average of the samples inoculated with the plant extract versus the positive control.

\begin{tabular}{ccccccc}
\hline & $\begin{array}{c}\text { Sample } \\
\text { time (h) }\end{array}$ & $\begin{array}{c}\text { Positive } \\
\text { Control }\end{array}$ & Sample 1 & Sample 2 & $\begin{array}{c}\text { Average of } \\
\text { 2 samples }\end{array}$ & $\begin{array}{c}\text { Percentage variation respect } \\
\text { to the positive control }\end{array}$ \\
\hline $\begin{array}{c}\text { Concentration of } \\
\text { microorganism with } \\
\text { the extract }\end{array}$ & 0 & $8.20 \times 10^{6}$ & $4.25 \times 10^{6}$ & $6.20 \times 10^{6}$ & $5.23 \times 10^{6}$ & $-36.22 \%$ \\
\hline $\begin{array}{c}\text { Addition of extract } \\
\text { on biofilm formed }\end{array}$ & 24 & $1.68 \times 10^{8}$ & $8.80 \times 10^{7}$ & $6.76 \times 10^{7}$ & $7.78 \times 10^{7}$ & $-53.69 \%$ \\
\hline
\end{tabular}


Table 4. Counts corresponding to the metal surface used in the snails farm. The results are expressed in CFU/total area sampled, and the percentage variation of the average of the samples inoculated with the plant extract versus the positive control.

\begin{tabular}{cccccccc}
\hline & $\begin{array}{c}\text { Sample } \\
\text { time (h) }\end{array}$ & $\begin{array}{c}\text { Positive } \\
\text { Control }\end{array}$ & Sample 1 & Sample 2 & Sample 3 & $\begin{array}{c}\text { Average } \\
\text { of 3 samples }\end{array}$ & $\begin{array}{c}\text { Percentage variation } \\
\text { respect to the } \\
\text { positive control }\end{array}$ \\
\hline $\begin{array}{c}\text { Concentration of } \\
\text { microorganism }\end{array}$ & 0 & $>1 \times 10^{5}$ & $>1 \times 10^{5}$ & $>1 \times 10^{5}$ & $>1 \times 10^{5}$ & $>1 \times 10^{5}$ & N.A. \\
$\begin{array}{c}\text { with the extract } \\
\text { Nition of extract }\end{array}$ & 24 & $2.72 \times 10^{4}$ & $1.17 \times 10^{4}$ & $1.39 \times 10^{4}$ & $1.34 \times 10^{4}$ & $1.30 \times 10^{4}$ & $-52.21 \%$ \\
\hline $\begin{array}{c}\text { Addiofilm formed } \\
\text { on biofm }\end{array}$ & 48 & $9.00 \times 10^{2}$ & $2.33 \times 10^{2}$ & $8.00 \times 10^{2}$ & $5.00 \times 10^{2}$ & $5.11 \times 10^{2}$ & $-44.44 \%$ \\
\hline
\end{tabular}

Table 5. Counts corresponding to the surface of fabric used in the snail farm. The results are expressed in CFU/total area sampled, and the percentage variation of the average of the samples inoculated with the plant extract versus the positive control.

\begin{tabular}{cccccccc}
\hline & $\begin{array}{c}\text { Sample } \\
\text { time (h) }\end{array}$ & $\begin{array}{c}\text { Positive } \\
\text { Control }\end{array}$ & Sample 1 & Sample 2 & Sample 3 & $\begin{array}{c}\text { Average of } \\
\text { 3 samples }\end{array}$ & $\begin{array}{c}\text { Percentage variation } \\
\text { respect to the } \\
\text { positive control }\end{array}$ \\
\hline $\begin{array}{c}\text { Concentration of } \\
\text { microorganism } \\
\text { with the extract }\end{array}$ & 0 & $>1 \times 10^{5}$ & $>1 \times 10^{5}$ & $>1 \times 10^{5}$ & $>1 \times 10^{5}$ & $>1 \times 10^{5}$ & N.A. \\
\hline $\begin{array}{c}\text { Addition of extract } \\
\text { on biofilm formed }\end{array}$ & 24 & $1.43 \times 10^{3}$ & $1.63 \times 10^{3}$ & $1.20 \times 10^{3}$ & $1.97 \times 10^{3}$ & $1.60 \times 10^{3}$ & $11.89 \%$ \\
\hline
\end{tabular}

results are expressed in CFU/unit of total area and percentage of variation of the samples with respect to the positive control.

Viable counts on the metal surface provided by the snail farm for the test for determining the inhibitory capacity of biofilm formation, showed very high results at time $0 \mathrm{~h}$, which were expressed as counts greater than $10^{5}$ per unit area. In these counts it was impossible to determine the percentage reduction of the samples with respect to the positive control. At $24 \mathrm{~h}$ and $48 \mathrm{~h}$ time, the counts showed decreases of $52.21 \%$ and $44.44 \%$ respectively.

The test carried out to determine the ability of the extract to destroy biofilm formed on the same surface, showed a decrease in the number of viables of $62.45 \%$ at initial time, and an increase of $133.63 \%$ at time $24 \mathrm{~h}$.

\subsection{Cloth Material Supplied by the Snail Farm}

The results of the fabric area used in the snail farm are shown in Table 5. The results are expressed in CFU/unit of total area and percentage of variation of the samples with respect to the positive control.

The results obtained in the test for determining the inhibitory capacity of biofilm formation, show counts above the detection limit of the technique at initial time, which were expressed as $>10^{5} \mathrm{CFU} /$ surface sampled. During this time, it was impossible to determine a percentage reduction of the samples with respect to the positive control. At $24 \mathrm{~h}$ and $48 \mathrm{~h}$ time, the counts showed increases of 
$11.89 \%$ and $60.86 \%$ respectively.

The test carried out to determine the capacity of the extract to destroy the biofilm formed showed a decrease in the number of viables of $22.78 \%$ at the initial time, and an increase of $45.88 \%$ at the $24 \mathrm{~h}$ time.

Considerable microorganism counts were not obtained in any of the portions of materials tested as negative controls.

\section{Discussion}

The way of life of some bacteria, in which the structured biofilm structure is being formed, together with the recent increase in the last years of resistance to antibiotics, made the treatment of diseases caused by bacteria is very difficult [15]. On many occasions, biofilm bacterial growth gives bacteria greater resistance to physical or chemical agents such as antimicrobials [16] [17]. Because of this, several studies have recently been conducted to demonstrate the potential antibiotic effect of certain molecules, including various quorum detection inhibitors in $S$. aureus and other microorganisms [15] [18] or of certain probiotic strains [19].

Many of the tested quorum-inhibiting molecules tested have been found to be modified to prevent biofilm formation. Even so, It has been seen that after its application on formed biofilm, the results are not as effective [15] [18]. Certain probiotic strains have also been found to be determined to inhibit the biofilm formation of $S$. aureus [19].

In this study, the potential of wheat extract to inhibit the formation of a biofilm or destroy an already formed biofilm of $S$. aureus, which is known for its biofilm-forming capacity, has been evaluated [3]. Other studies have highlighted the possible biotechnological applications of wheat extract [14] and its inhibitory effect on microbial films [1].

Different surface materials (PVC surface, metallic surface, cloth surface and drinking water channelling material) used in snail and poultry production farms have been evaluated, in which biofilm of $S$. aureus has been formed in vitro and, subsequently wheat extract has been added to evaluate its function.

According to the results obtained in this study, a better adherence and biofilm formation of Staphylococcus aureus can be considered in the water channelling material supplied by the poultry farm compared to any other surface supplied by the snail farm.

In the PVC material provided by the snail farm, the microorganism has a moderate adherence and biofilm formation capacity, since it starts from values of the order of $10^{4}$ at the initial time for the test for determining the inhibitory capacity of biofilm formation, and of the order of $10^{3}$ at initial time for the test of the lytic capacity on biofilm formed. These values were reduced to orders of $10^{2}$ CFU to $10^{1} \mathrm{CFU}$ after 24 or 48 hours of incubation. Table 1 shows the percentage reduction in the number of viables in each test after 24 and 48 hours of incubation, suggesting an extract efficiency of around 10\% for the inhibition test and $60 \%$ for the lysis test of the extract on said surface. Previously, high percen- 
tages of reduction by wheat extract were also reported [1].

In the material from the poultry farm, the results indicate greater effectiveness of the plant extract at high concentrations of microorganism in both tests, presenting for the inhibition test a viable decrease of more than $85 \%$ in both liquid and surface after $48 \mathrm{~h}$ after of inoculation. In the test to test the ability to destroy the biofilm formed, the percentage reduction of viables was greater than $95 \%$ after 24 hours after inoculation of the extract on the biofilm formed on the inner surface (Table 3). Therefore, it can be indicated that the extract has high efficiency on this surface in both tests tested.

Both in the metallic material provided by the snail farm and in the cloth material, the microorganism has a low adherence capacity, since it has counted at initial time outside the detection range with the technique used, which decrease to residual values, in the order of $10^{1} \mathrm{CFU}$ to $10^{2} \mathrm{CFU}$ per unit area at 24 and 48 $\mathrm{h}$, which are also not indicators of biofilm formation. Therefore, it was impossible to determine the efficacy of the plant extract on these surfaces.

\section{Conflicts of Interest}

The authors declare no conflicts of interest regarding the publication of this paper.

\section{References}

[1] González-Ortiz, G., Quarles Van Ufford, H.C., Halkes, S.B.A., Cerdà-Cuéllar, M., Beukelman, C.J., Pieters, R.J. and Martín-Orue, S.M. (2014) New Properties of Wheat Bran: Anti-Biofilm Activity and Interference with Bacteria Quorum-Sensing Systems. Environmental Microbiology, 16, 1346-1353. https://doi.org/10.1111/1462-2920.12441

[2] Gaggìa, F., Mattarelli, P. and Biavati, B. (2010) Probiotics and Prebiotics in Animal Feeding for Safe Food Production. International Journal of Food Microbiology, 141, S15-S28. https://doi.org/10.1016/j.ijfoodmicro.2010.02.031

[3] Otto, M. (2013) Staphylococcal Infections: Mechanisms of Biofilm Maturation and Detachment as Critical Determinants of Pathogenicity. Annual Review of Medicine, 64, 175-188. https://doi.org/10.1146/annurev-med-042711-140023

[4] Thacker, P.A. (2013) Alternatives to Antibiotics as Growth Promoters for Use in Swine Production: A Review. Journal of Animal Science and Biotechnology, 4, Article No. 35. https://doi.org/10.1186/2049-1891-4-35

[5] Brackman, G. and Coenye, T. (2014) Quorum Sensing Inhibitors as Anti-Biofilm Agents. Current Pharmaceutical Design, 21, 5-11. https://doi.org/10.2174/1381612820666140905114627

[6] Costa, J.C.M., de Freitas Espeschit, I., Pieri, F.A., dos Anjos Benjamin, L. and Moreira, M.A.S. (2012) Increased Production of Biofilms by Escherichia Coli in the Presence of Enrofloxacin. Veterinary Microbiology, 160, 488-490. https://doi.org/10.1016/j.vetmic.2012.05.036

[7] Gavin, L. (2012) Microbial Biofilms: Current Research and Applications. Caister Academic Press, Poole, UK.

[8] Sharon, N. (2006) Carbohydrates as Future Anti-Adhesion Drugs for Infectious Diseases. Biochimica et Biophysica Acta-General Subjects, 1760, 527-537. 
https://doi.org/10.1016/j.bbagen.2005.12.008

[9] Lane, J.A., Mehra, R.K., Carrington, S.D. and Hickey, R.M. (2010) The Food Glycome: A Source of Protection against Pathogen Colonization in the Gastrointestinal Tract. International Journal of Food Microbiology, 142, 1-13. https://doi.org/10.1016/j.ijfoodmicro.2010.05.027

[10] González, G. (2013) Natural Sources against Veterinary Pathogens: Evaluation of the Anti-Adhesiveand Anti-Biofilm Activity of Wheat Bran. Thesis, Universitat Autònoma de Barcelona, Cerdanyola del Vallès, Spain.

[11] Dong, Y.-H., Wang, L.-H. and Zhang, L.-H. (2007) Quorum-Quenching Microbial Infections: Mechanisms and Implications. Philosophical Transactions of the Royal Society B: Biological Sciences, 362, 1201-1211. https://doi.org/10.1098/rstb.2007.2045

[12] Boyen, F., Eeckhaut, V., Van Immerseel, F., Pasmans, F., Ducatelle, R. and Haesebrouck, F. (2009) Quorum Sensing in Veterinary Pathogens: Mechanisms, Clinical Importance and Future Perspectives. Veterinary Microbiology, 135, 187-195. https://doi.org/10.1016/j.vetmic.2008.12.025

[13] Molist, F., de Segura, G.A., Pérez, J.F., Bhandari, S.K., Krause, D.O. and Nyachoti, C.M. (2010) Effect of Wheat Bran on the Health and Performance of Weaned Pigs Challenged with Escherichia Coli K88 ${ }^{+}$. Livestock Science, 133, 214-217. https://doi.org/10.1016/j.livsci.2010.06.067

[14] Mohsin Javed, M., Zahoor, S., Shafaat, S., Mehmooda, I., Gul, A., Rasheed, H., Bukhari, S.A.I., Nauman Aftab, M. and Ikram-ul-Haq (2012) Wheat Bran as a Brown Gold: Nutritious Value and Its Biotechnological Applications. African Journal of Microbiology Research, 6, 724-733. https://doi.org/10.5897/AJMRX11.035

[15] Brackman, G., Cos, P., Maes, L., Nelis, H.J. and Coenye, T. (2011) Quorum Sensing Inhibitors Increase the Susceptibility of Bacterial Biofilms to Antibiotics in Vitro and in Vivo. Antimicrobial Agents and Chemotherapy, 55, 2655-2661. https://doi.org/10.1128/AAC.00045-11

[16] Bhardwaj, A.K., Vinothkumar, K. and Rajpara, N. (2013) Bacterial Quorum Sensing Inhibitors: Attractive Alternatives for Control of Infectious Pathogens Showing Multiple Drug Resistance. Recent Patents on Anti-infective Drug Discovery, 8, 68-83. https://doi.org/10.2174/1574891X11308010012

[17] Jiang, Q., Chen, J., Yang, C., Yin, Y., Yao, K. and Song, D. (2019) Quorum Sensing: A Prospective Therapeutic Target for Bacterial Diseases. BioMed Research International, 2019, Article ID: 2015978. https://doi.org/10.1155/2019/2015978

[18] Chen, H., Wubbolts, R.W., Haagsman, H.P. and Veldhuizen, E.J.A. (2018) Inhibition and Eradication of Pseudomonas aeruginosa Biofilms by Host Defence Peptides. Scientific Reports, 8, Article No. 10466. https://doi.org/10.1038/s41598-018-28842-8

[19] Vera Garcia, R.E. (2016) Microbiología del caracol Helix aspersa Muller. Aplicaciones biotecnológicas para su mejoramiento sanitario con impacto en su comercialización. Universitat Autònoma de Barcelona, Cerdanyola del Vallès, Spain. 